\title{
Nonmonotonic Rule Systems on Top of Ontology Layers
}

\author{
Grigoris Antoniou \\ Dept. of Computer Science, University of Bremen, Germany \\ ga@tzi.de
}

\begin{abstract}
The development of the Semantic Web proceeds in layers. Currently the most advanced layer that has reached maturity is the ontology layer, in the from of the DAML+OIL language which corresponds to a rich description logic. The next step will be the the realization of logical rule systems on top of the ontology layer.

Computationally simple nonmonotonic rule systems show promise to play an important role in electronic commerce on the Semantic Web. In this paper we outline how nonmonotonic rule systems in the form of defeasible reasoning, can be built on top of description logics.
\end{abstract}

\section{Motivation and Overview}

The Semantic Web initiative 17] promises to improve dramatically the World Wide Web, and in doing so, to have significant impact on the way information is exchanged and business is conducted. The main idea is to use machine processable data and knowledge.

The development of the semantic web proceeds in layers, each layer being on top of lower layers. At present, the highest layer that has reached sufficient maturity is the ontology layer in the form of the DAML+OIL language 4. DAML+OIL was designed to be sufficiently rich to be useful in applications, while being simple enough to allow for efficient reasoning support. In fact, it corresponds to an expressive description logic. Stated another way, the realization of the ontology layer could draw benefits from extensive previous work on description logics [31211, both with regard to clear semantics and efficient reasoning support.

The next step in the development of the semantic web core will be the realization of the logic and proof layers. These layers will be built on top of ontology languages, and will offer enhanced representation and reasoning capabilities. A key ingredient of these layers will be rules, which look likely to become an action focus of the W3C. Monotonic rule systems are well known and widely in use. Seen as a subset of predicate logic (Horn logic), they are orthogonal to description logics: none is proper subset of the other. The realization of a monotonic rule layer on top of the ontology layer can draw on work on hybrid reasoning, combining description logics with Horn logic, or at least Datalog [12,67].

But there exist other kinds of rule systems that are nonmonotonic. Such systems are important in practice because they can deal with inconsistencies in a 
declarative way, and because they model naturally phenomena like exceptions and priorities. In the past few years, such systems have attracted significant attention in the nonmonotonic reasoning community, e.g. courteous logic programs [9] and defeasible logics 141. Their use in various application domains has been advocated, including the modelling of regulations and business rules [10], modelling of contracts [10, legal reasoning [15] and agent negotiations 8]. In fact, defeasible reasoning (in the form of courteous logic programs [9]) provides the foundation for IBM's Business Rules Markup Language and for current W3C activities on rules. Therefore defeasible reasoning is arguably one of the most successful subarea in nonmonotonic reasoning as far as applications and integration to mainstream IT is concerned.

One important advantage of such systems is their focus on implementability and their low computational complexity [13. So seen, it makes sense to study the integration of description logics with defeasible reasoning, since both share a focus on efficiency. The integration of nonmonotonic rule systems with description logic based ontologies can serve two purposes: (1) Enhanced reasoning capabilities may be used to express richer ontological knowledge. For example, defeasible ontologies can be built, an idea that appears reasonable in the legal domain. (2) Rule- based systems define ontology-based applications using vocabulary defined in description logic. This idea is compatible with significant work on hybrid reasoning, e.g. [12].

In this paper we concentrate on the second approach, and we will study defeasible reasoning [14] running on top of description logics. Our task is more complex than the integration of Horn rules with description logics because of the following observation: both Horn logic and description logics are subsets of predicate logic. Therefore semantically there is no difficulty at all, and the focus of work on hybrid reasoning has been on efficient algorithms and limits of computability. However nonmonotonic rules are not a subset of predicate logic, and we need to define the semantics of such a combination, too. On the other hand, because nonmonotonic rules with variables are interpreted as schemas, their integration with description logics does not have difficulties encountered in other works on hybrid reasoning with regard to instantiation [6].

The situation gets more complicated if concept and role predicates are allowed in the heads of rules as well. We intend to study such tighter integration in the future. Also we have not addressed issues of language design. In this sense, this paper reports on ongoing work. However the preliminary results are still significant, because they show for the first time how, in principle, a defeasible logic and inference layer can be realised on top of the ontology layer.

\section{Knowledge Bases}

A knowledge base $K=(T, F, R,>)$ consists of

- A terminology $T$, defined in a description logic.

- A set $F$ of facts. Each fact has the form $p\left(a_{1}, \ldots, a_{m}\right)$, where $p$ is a predicate, and $a_{1}, \ldots, a_{m}$ are constants. $F$ is the disjoint union of a set $F_{T}$ of facts 
with a concept or role predicate, and a remainder $F_{O}$. Predicates that are not concept and role predicates are called ordinary.

- A set $R$ of rules

$$
L_{1}, \ldots, L_{n} \Rightarrow L
$$

such that all $L$ and $L_{i}$ are literals $p\left(a_{1}, \ldots, a_{m}\right)$ or $\neg p\left(a_{1}, \ldots, a_{m}\right)$, with constants $a_{1}, \ldots, a_{m}$ and a concept, role or ordinary predicate $p$. Additionally, the predicate of $L$ must be an ordinary predicate. $\left\{L_{1}, \ldots, L_{n}\right\}$ is the set of antecedents of the rule $r$, denoted $A(r)$. And $L$ is called the head (or consequent) of $r$, denoted $C(r)$.

- an acyclic relation $>$ on $R$.

Now we make a number of remarks.

1. Rules with variables are interpreted as schemas: they represent the set of their ground instances. This interpretation is standard in many nonmonotonic reasoning approaches, among others in default logic [16] and defeasible logics 14 .

2. The logical language does not have function symbols, thus the Herbrand universe is finite.

3. Concept and role predicates are not allowed to occur in facts, or the heads of rules. This design decision follows the idea that rules may not be used to derive ontological knowledge. All knowledge about concepts and roles is provided by the description logic component. The same idea was followed by other work on hybrid reasoning involving description logic and monotonic rules, e.g. 12. The motivation for such an approach, and its relevance to the semantic web initiative, were outlined in the introduction.

4. Defeasible logic usually offers strict rules, and sometimes defeaters, in addition. We have omitted defeaters here, because they can be simulated by other means [1]. And we have decided to omit strict rules, because typically they include taxonomical, or other kinds of certain knowledge. We assume that such knowledge will be included in the ontology, and treated by the description logic. Instead we have allowed a set of facts about ordinary predicates. If need be, strict rules can be easily added to our logical system.

Given a set $R$ of rules, $R[L]$ denotes the set of rules in $R$ with head $L$. In the following $\sim L$ denotes the complement of $L$, that is, $\sim L$ is $\neg L$ if $L$ is an atomic formula, and $\sim L$ is $L^{\prime}$ if $L$ is $\neg L$.

\section{Defeasible Reasoning Using Terminological Knowledge}

Given a rule $r$

$$
L_{1}, \ldots, L_{n} \Rightarrow L
$$

suppose $\left\{L_{1}, \ldots, L_{n}\right\}$ is partitioned into $\left\{L^{(1)}, \ldots, L^{(k)}\right\}$ and $\left\{L^{(k+1)}, \ldots, L^{(n)}\right\}$, such that the predicates of $\left\{L^{(1)}, \ldots, L^{(k)}\right\}$ are ordinary, and the predicates of $\left\{L^{(k+1)}, \ldots, L^{(n)}\right\}$ are concept or role predicates. Further suppose that $T \cup F \models$ $L^{(j)}$ for all $j \in\{k+1, \ldots, n\}$. Then the rule 


$$
L^{(1)} \ldots, L^{(k)} \Rightarrow L
$$

is called the reduct of $r$; otherwise the reduct is undefined. For a set $R$ of rules, $\operatorname{Red}(R)$ collects the reducts of all rules in $R$.

Now we briefly outline the proof theory. A conclusion of a defeasible theory $D$ is a signed tagged literal. Here we use only one tag, $\partial$, which denotes defeasible provability. Also we use the signs + and - . A conclusion $+\partial L$ means that the literal $L$ is defeasibly provable in $D$. And $-\partial q$ means that we have proved that $q$ is not defeasibly provable in $D$.

A derivation (or proof) $P$ is a finite sequence of signed tagged literals. $P(1 . . i)$ denotes the first $i$ elements of this sequence. Now we proceed to give the inference conditions for $+\partial$ and $-\partial$. Let $L$ be a literal with an ordinary predicate (Here we define $\partial L$ only for such literals. A simple extension would be to derive $+\partial L$ iff $T \cup F \mid=L$, where the predicate of $L$ is a concept or role predicate).

$+\partial$ : If $P(i+1)=+\partial L$ then either

(1) $L \in F$ or

(2) (2.1) $\exists r \in \operatorname{Red}(R)[L] \forall L^{\prime} \in A(r):+\partial L^{\prime} \in P(1 . . i)$

$(2.2) \sim L \notin F$ and

(2.3) $\forall s \in \operatorname{Red}(R)[\sim L]$ either

$$
\begin{gathered}
\text { (2.3.1) } \exists L^{\prime} \in A(s):-\partial L^{\prime} \in P(1 . . i) \text { or } \\
\text { (2.3.2) } \exists t \in \operatorname{Red}(R)[L] \forall L^{\prime} \in A(t) \\
+\partial L^{\prime} \in P(1 . . i) \text { and } t>s .
\end{gathered}
$$

Let us illustrate this definition. To show that $L$ is provable defeasibly we have two choices: (1) We show that $L$ is a fact; or (2) we need to argue using rules. In particular, we require that there must be a rule reduct with head $L$ which can be applied (2.1). But now we need to consider possible "counterattacks", that is, reasoning chains in support of $\sim L$. To be more specific: to prove $L$ using rule reducts we must show that $\sim L$ is not a fact (2.2). Also (2.3) we must consider the set of all rule reducts which are not known to be inapplicable and which have head $\sim L$. Essentially each such rule $s$ attacks the conclusion $L$. For $L$ to be provable, each such rule $s$ must have been established as non-applicable (2.3.1). Altenratively there must be a rule reduct with head $L$ stronger than the attacking rule (2.3.2).

The inference condition $-\partial$ is the so-called strong negation of + (de Morgan is applied, and + and - are interchanged).

Implementation will involve interleaving of description logic reasoners and defeasible reasoners. Derivability of antecedents with concept or role predicates will be checked by a description logic reasoner, the remainder is treated as specified in the inference conditions above.

Example 1. Imagine an online store which has organised its stock according to an ontology. Among others, the ontology contains the information physicsBook $\sqsubseteq$ scientificBook $\sqsubseteq$ book. The pricing policy of the store is written in defeasible logic, and might include the following information (scientific books get a special $5 \%$ discount). 
$r_{1}: \Rightarrow \neg \operatorname{discount}(X, Y, Z)$

$\left.r_{2}: \operatorname{scientificBook}(X) \Rightarrow X, Y, 5 \%\right)$

(where $X$ denotes an article and $Y$ a customer). The item with id 93215 is stored in the corporate data base as a physics book:

physicsBook $(93215)$

Putting all this information together, we can derive $+\partial \operatorname{discount}(93215, Y, 5 \%)$.

\section{References}

1. G. Antoniou, D. Billington, G. Governatori and M.J. Maher. Representation Results for Defeasible Logic. ACM Transactions on Computational Logic 2,2 (2001): $255-287$.

2. F. Baader and B. Hollunder. A terminological knowledge representation system with complete inference algorithm. In Proc. Workshop on Processing Declarative Knowledge, LNAI, Springer 1991, 67-86.

3. M. Buchheit, F. Donini and A. Schaerf. Decidable Reasoning in terminological knowledge representation systems. Journal of Artificial Intelligence Research 1 (1993): 109-138.

4. D. Connolly et al. DAML+OIL (March 2001) Reference Description. http://www.w3.org/TR/daml+oil-reference.

5. F. Donini, M. Lenzerini, D. Nardi and A. Schaerf. Reasoning in Description Logics. In G.Brewka (ed): Principles of Knowledge Representation and Reasoning, Studies in Logic, Language and Information, CLSI Publications 1996, 193-238.

6. F. Donini, M. Lenzerini, D. Nardi and A. Schaerf. A hybrid system with datalog and concept languages. In E. Ardizzone, S. Gaglio, F. Sorbello (eds): Trends in Artificial Intelligence, LNAI 549, Springer 1991, 88-97.

7. A. Frisch (ed). Workshop Notes of the AAAI Fall Symposium on Principles of Hybrid Reasoning. AAAI Press 1991.

8. G. Governatori, A. ter Hofstede and P. Oaks. Defeasible Logic for Automated Negotiation. Proc. Collecter'2000.

9. B. Grosof. Prioritized conflict handling for logic programs. In Proc. International Logic Programming Symposium, MIT Press 1997, 197-211.

10. B. Grosof, Y. Lambrou and H. Chan. A Declarative Approach to Business Rules in Contracts: Courteous Logic Programs in XML. In Proc. 1st ACM Conference on Electronic Commerce, ACM 1999.

11. I. Horrocks and U. Sattler. Ontology reasoning in the SHOQ(D) description logic. In Proc. of the 17th Int. Joint Conf. on Artificial Intelligence (IJCAI-01), Morgan Kaufmann 2001, 199-204.

12. A. Levy and M-C. Rousset. CARIN: A Representation Language Combining Horn rules and Description Logics. Artificial Intelligence 104(1-2), 1998, 165-209.

13. M.J. Maher. Propositional Defeasible Logic has Linear Complexity. Theory and Practice of Logic Programming, 1,6 (2001): 691-711.

14. D. Nute. Defeasible Logic. In D.M. Gabbay, C.J. Hogger and J.A. Robinson (eds.): Handbook of Logic in Artificial Intelligence and Logic Programming Vol. 3, Oxford University Press 1994, 353-395.

15. H. Prakken. Logical Tools for Modelling Legal Argument: A Study of Defeasible Reasoning in Law. Kluwer Academic Publishers 1997.

16. R. Reiter. A Logic for Default Reasoning. Artificial Intelligence 13(1980): 81-132.

17. www.w3.org/2001/sw/ 